\title{
Trasplante renal sobre prótesis aortobifemoral
}

\author{
Aguilera Tubet C, Gutiérrez Baños JL, Portillo Martín JA, Valle Del Schaan JI, \\ Correas Gómez MA, Roca Edreira A.
}

Servicio de Urología. Hospital Universitario Marqués de Valdecilla. Santander, Cantabria.

Actas Urol Esp. 2008;32(3):341-344

\section{RESUMEN}

TRASPLANTE RENAL SOBRE PROTESIS AORTOBIFEMORAL

Introducción: Cada vez es más frecuente en la práctica clínica, la existencia de pacientes en insuficiencia renal terminal subsidiarios de trasplante renal que han recibido con anterioridad una cirugía protésica de revascularización. Hasta hace poco tiempo, estos pacientes eran desestimados como candidatos a trasplante renal. Contamos aquí nuestra experiencia en cinco casos.

Material y métodos: De un total de 1483 trasplantes renales, 5 eran pacientes portadores de prótesis aorto-bifemoral (2 por aneurisma y 3 por enfermedad oclusiva). Revisamos las características, evolución y complicaciones de estos pacientes.

Resultados: El injerto vascular se había colocado entre 6 meses y 16 años antes del trasplante. La técnica quirúrgica del trasplante fue la clásica, anastomosando la arteria renal al injerto vascular. Complicaciones quirúrgicas fueron; 1 trombosis de la arteria renal que precisó trombectomía y 1 estenosis ureterovesical. 2 pacientes fallecieron con injerto funcionante por neumonía a los 6 meses y 7 años, 3 viven con injerto funcionante a los 7 meses, 3 y 7 años. Durante la evolución aparecieron otras complicaciones vasculares no relacionadas con el trasplante.

Conclusión: El trasplante renal sobre prótesis aorto-bifemoral es una opción válida en pacientes seleccionados.

Palabras clave: Trasplante renal. Prótesis Insuficiencia renal.

\section{ABSTRACT}

RENAL TRANSPLANTATION IN PATIENTS WITH A VASCULAR AORTOBIFEMORAL PROSTHESIS

Introduction: Nowadays, it is much more common in end stage renal disease patients with vascular grafts, to be kidney transplant candidates. We expose our experience in five cases.

Material and methods: Of all 1,483 kidneys transplanted in our center, 5 recipients had a previous aortobifemoral bypass ( 2 due to abdominal aortic aneurysm, and 3 due to vascular occlusive disease). We review the clinical features, outcome and complications in these patients.

Results: The vascular surgery was done 6 months to 16 years prior to transplantation. The renal transplant was done in iliac fossa with arterial anastomosis to the vascular graft. Surgical complications were: 1 renal artery thrombosis that was treated with thrombectomy, and 1 stricture at the ureterovesical junction. 2 patients dead at 6 months and 7 years with a functioning allograft and 3 patients live with functional allograft at 7 months, 3 years and 7 years.

Conclusion: Kidney transplantation may be successful in selected patients with aortobifemoral bypass. Keywords: Renal grafts. Prosthesis. Renal failure. 
$\mathrm{E}^{\prime}$ n la actualidad los grupos que realizan trasplante renal se encuentran con pacientes que en otros tiempos serían descartados como receptores de injerto renal, debido a su edad elevada o a la coincidencia en ellos de varios factores cardiovasculares que dificultan y ensombrecen la cirugía y los resultados funcionales de dichos trasplantes. Así es que, el aumento de la edad media de los pacientes en lista de espera de trasplante renal junto con las características que definen la enfermedad renal terminal provocan un aumento en la prevalencia de patología arterial periférica, principalmente obstructiva, en este grupo de pacientes ${ }^{1,2}$. De esta manera, nos encontramos con candidatos a un trasplante renal que han recibido una cirugía previa de revascularización como es el bypass aorto-bifemoral, pacientes portadores de un injerto renal que precisan con posterioridad una cirugía vascular protésica, o bien, pacientes en que se plantea la cirugía de forma simultánea.

\section{MATERIAL Y MÉTODOS}

Entre enero de 1975 y diciembre de 2006, se han realizado 1.483 trasplantes en nuestro hospital, de estos, 5 pacientes portaban con anterioridad una prótesis aorto-bifemoral por patología arterial periférica, implantándose el injerto renal sobre esta.

Realizamos estudio retrospectivo, revisando: causa de la insuficiencia renal, causa del implante aorto-bifemoral, tiempo entre las dos cirugías, tipo de anastomosis vasculares, evolución del injerto y paciente y complicaciones quirúrgicas.

\section{RESULTADOS}

En los cinco casos se trataba de pacientes varones y su edad media en el momento del trasplante era de 56,8 años (con un rango de 43 a 64 años). La causa de la insuficiencia renal fue enfermedad vascular renal sólo en uno de los pacientes, y poliquistosis hepato-renal, nefropatía diabética y esclerosis renal en los demás, siendo indeterminada en un caso. La cirugía del bypass se había efectuado entre 6 meses y 16 años antes de la cirugía del trasplante y la indicación había sido enfermedad aneurismática en 2 pacientes y obstructiva en 3 .

Todos los injertos procedían de donante cadáver, con un tiempo de isquemia fría de 16 a 25 horas, con una media de 20,4 horas.
La técnica quirúrgica del trasplante fue la clásica, por vía extraperitoneal y en fosa ilíaca en los cinco casos, con anastomosis vascular terminolateral a la prótesis y a la vena ilíaca común o externa. La técnica de ureteroneocistostomía fue siempre extravesical y tutorizada con catéter ureteral tipo doble "J" (Fig. 1).

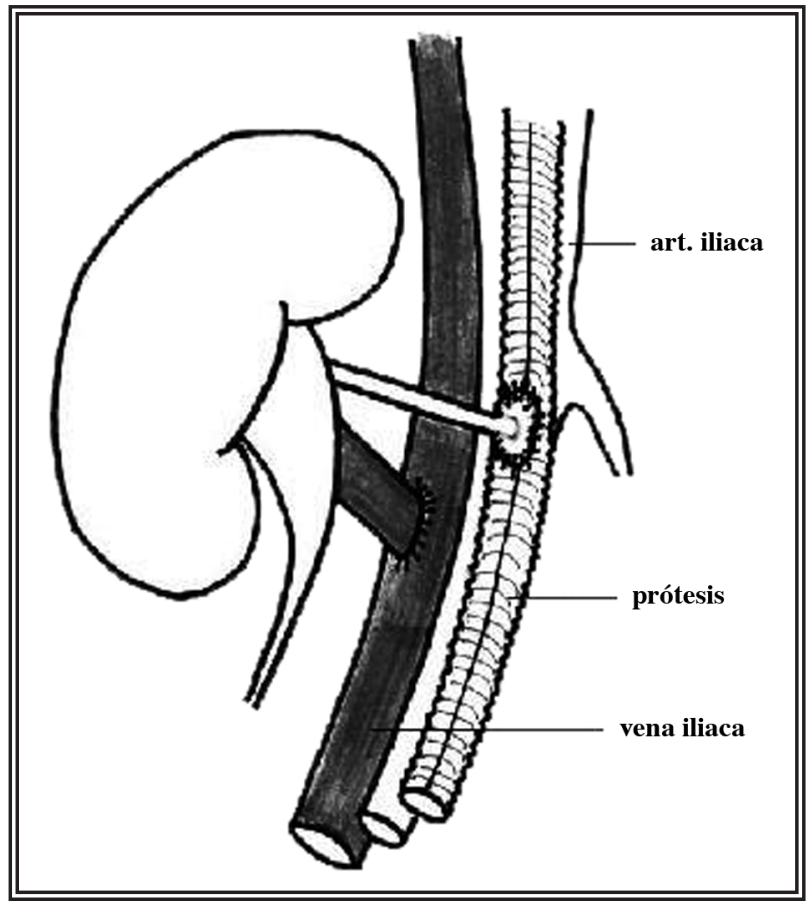

FIGURA 1

En un caso se produjo una trombosis de la arteria del injerto que precisó trombectomía. En este mismo paciente se produjo una estenosis de la anastomosis ureterovesical que no pudo ser resuelta mediante dilataciones endoscópicas por lo que se reimplantó, recidivando la estenosis a los 4 meses, realizando en este momento una anastomosis pelvis-pelvis que resolvió el problema. Siete años después, este paciente permanece vivo y con el injerto funcionante (Tabla 1).

Dos pacientes fallecieron por sepsis secundaria a neumonía con el injerto funcionante, 6 meses y 7 años tras la realización del trasplante.

En resumen, tres pacientes permanecen vivos, con el injerto funcionante tras 7 meses, 3 años y 7 años, con cifras de creatinina que oscilan entre $1 \mathrm{y}$ $1,6 \mathrm{mg} / \mathrm{dl}$.

Durante la evolución postoperatoria acontecieron otras complicaciones de origen vascular: cardio- 
Tabla 1. Complicaciones quirúrgicas

\begin{tabular}{ll}
\hline Complicaciones inmediatas & Complicaciones tardias \\
\hline $\begin{array}{l}1 \text { hematoma lecho } \\
\text { (observación) }\end{array}$ & $\begin{array}{l}1 \text { estenosis anastomosis } \\
\text { ureterovesical }\end{array}$ \\
1 trombosis arteria injerto & \\
(trombectomía) & \\
\hline
\end{tabular}

patía isquémica y amputación de extremidad inferior en el mismo paciente a los dos años del trasplante, accidente vascular cerebral a los cinco años y rotura de un aneurisma iliofemoral a los dos años del trasplante en otro paciente.

\section{DISCUSIÓN}

Es un hecho bien establecido que la edad media de los pacientes candidatos a trasplante renal aumenta. Esto se une a una mayor prevalencia de diabetes e hipertensión, que junto a la hemodiálisis, aumentan la incidencia de patología vascular en este grupo de pacientes ${ }^{1}$. De esta manera, nos encontramos cada vez con un mayor número de pacientes con insuficiencia renal terminal que ya han recibido una cirugía protésica de revascularización, al igual que pacientes trasplantados que la necesitarán en un futuro. Asímismo, nos podemos encontrar con pacientes subsidiarios de trasplante renal que precisan además una cirugía de revascularización simultánea.

El primer caso de trasplante renal en un paciente con prótesis vascular previa es reportado en la literatura por Sterioff et al. ${ }^{3}$ en 1974.

Varios autores han comunicado con posterioridad sus series. Brekke et $a{ }^{4}$ presentan 20 casos con cirugía vascular reconstructiva como preparación para el trasplante. Gouny et al. ${ }^{5}$ publican una serie de 5 pacientes con cirugía vascular reconstructiva y trasplante renal simultaneo. Van der Vliet ${ }^{6}$ comunica 13 enfermos en que la reconstrucción vascular y el trasplante fueron simultáneos y 7 en que fueron diferidos.

En el caso en que el trasplante renal se realice con posterioridad a la cirugía de reconstrucción vascular, la mayor parte de los autores coinciden en que la dificultad radica en la disección de la vena iliaca por la fibrosis que se forma en torno a la prótesis vascular, debida a la fibrosis retroperitoneal producida por el aneurisma y a la reacción tisular al material sintético de la prótesis ${ }^{5,7}$. En casos en que esta se encuentre muy dificultada y exista riesgo de lesión de la misma, la anastomosis venosa se puede realizar directamente a la vena cava ${ }^{5,8}$. TalbotWright et al. en estos casos abogan por la realización de un trasplante renal ortotópico ${ }^{9}$.

En nuestros cinco casos, se pudo constatar la mayor dificultad en la disección de la vena ilíaca, pero en todos los casos fue posible la anastomosis venosa a la misma.

Dos de los requisitos fundamentales de las prótesis vasculares son la impermeabilidad a la fuga de sangre y su no trombogenicidad. Otro requisito muy importante en los pacientes que van a recibir un trasplante renal y que por lo tanto están inmunodeprimidos, es su resistencia a la infección. Teniendo en cuenta esto último, la prótesis más adecuada parece ser la de PTFE, ya que por su superficie más suave dificulta la adhesión bacteriana. Además, tiene mayor fuerza tensil en la anastomosis, menor depósito plaquetario y menor activación del complemento con respecto al Dacron ${ }^{10}$. Uno de sus inconvenientes es la tendencia al sangrado por los orificios de paso de la aguja de sutura, por eso se recomienda que el material de sutura sea de aguja pequeña, calibre fino y del mismo material, para minimizar el orificio que deja la sutura sobre la prótesis y con ello el sangrado. En el caso de que este ocurra, son útiles materiales hemostáticos y sellantes locales.

Otro grupo de pacientes son aquellos con insuficiencia renal terminal subsidiarios de trasplante renal pero que presentan patología vascular severa del sector aortoilíaco. Antiguamente, estos pacientes eran desestimados como candidatos, pero actualmente se realiza una cirugía de revascularización y el trasplante. La controversia está en la posibilidad de efectuar la cirugía de forma simultánea o bien diferida en el tiempo, realizando la cirugía de revascularización entre 6 y 12 semanas antes del trasplante ${ }^{5}$. Wright et al. creen que existe un claro beneficio al realizar la cirugía de forma simultánea, argumentando que no existen dificultades técnicas asociadas a la reoperación, los pacientes tendrán un riñón con una buena función al final de la intervención, lo cual facilita el manejo de líquidos y electrolitos durante el postoperatorio, los costes y el tiempo de hospitalización son menores, existe sólo un riesgo anestésico, y por último, el sangrado por los intersticios de la prótesis parece ser menor en los pacientes con buena función renal que en los urémicos ${ }^{7}$. 
El otro tipo de situación clínica en que nos podemos encontrar, son pacientes que recibieron en su día un trasplante renal y que ahora precisan de una cirugía de revascularización ${ }^{8,11,12}$. Es una situación excepcional, pero que, con el aumento de la supervivencia de estos pacientes, tiende a ser más frecuente. No es objeto de este trabajo profundizar en sus particularidades, pero si queremos resaltar la necesidad de protección renal según la duración de la cirugía de revascularización y la función previa del injerto, mediante alguno de los métodos descritos hasta la fecha, siempre a criterio del cirujano.

$\mathrm{El}$ postoperatorio en estos pacientes puede verse complicado por la patología coronaria que puede estar subyacente en estos pacientes, la cual dificulta el manejo hemodinámica en el postoperatorio inmediato e este grupo de pacientes. De hecho, la principal causa de muerte en estos pacientes es de origen cardiovascular. Esto traduce la presencia de enfermedad vascular sistémica y una mayor prevalencia de arterioesclerosis coronaria en este grupo de pacientes ${ }^{5,13}$.

\section{CONCLUSIÓN}

El trasplante renal sobre prótesis aorto-bifemoral es una opción válida en pacientes seleccionados, que consigue unos resultados razonables de funcionalidad del injerto, mejorando la calidad de vida de los receptores.

\section{REFERENCIAS}

1. Rajagopalan S, Dellegrottaglie S, Furniss AL, Gillespie BW, Satayathum S, Lameire N, et al. Peripheral arterial disease in patients with end-stage renal disease: observations from the Dialysis Outcomes and Practice Patterns Study (DOPPS). Circulation. 2006;114(18):1914-1922.
2. Guerrero A, Montes R, Muñoz-Terol J, Gil-Peralta A, Toro J, Naranjo M, et al. Peripheral arterial disease in patients with stages IV and V chronic renal failure. Nephrol Dial Transplant. 2006;21(12):3525-3231.

3. Sterioff S, Zachary JB, Williams GM. Dacron vascular grafts in renal transplant patients. Am J Surg. 1974;127(5): 525-528.

4. Brekke IB, Lien B, Sødal G, Jakobsen A, Bentdal O, Pfeffer P, et al. Aortoiliac reconstruction in preparation for renal transplantation. Transpl Int. 1993;6(3):161-163.

5. Gouny P, Lenot B, Decaix B, Rondeau E, Kitzis M, Lacave R, et al. Aortoiliac surgery and kidney transplantation. Ann Vasc Surg. 1991;5(1):26-31.

6. Van der Vliet JA, Naafs DB, van Bockel JH, Kootstra G, Boll AP, Barendregt WB, et al. Fate of renal allografts connected to vascular protheses. Clin Transplantation. 1996;10(2):199-202.

7. Wright JG, Tesi RJ, Massop DW, Henry ML, Durham JR, Ferguson RM, et al. Safety of simultaneous aortic reconstruction and renal transplantation. Am J Surg. 1991;162(2):126-130.

8. Pittaluga P, Hassen-Khodja R, Cassuto-Viguier E, Batt M, Declemy S, Bariseel H, et al. Aortoiliac reconstruction and kidney transplantation: a multicenter study. Ann Vasc Surg. 1998; 12(6):529-536.

9. Talbot-Wright R, Carretero González P, Gutiérrez del Pozo R, Alcocer García JA. Trasplante renal en receptores con patología vascular compleja. González Martín y García Buitrón eds: Trasplante renal. Madrid (España). Ediciones Aula Médica S.A. 2000. 163-177.

10. Brewster RB. Prosthetic grafts. Rutherford: Vascular Surgery (4 ${ }^{\text {a }}$ Ed.). 1995.

11. Lacombe M. Aortoiliac surgery in renal transplant patients. J Vasc Surg. 1991;13(5):712-718.

12. Panneton JM, Gloviczki P, Canton LG, Bower TC, Chow MS, Pairolero PC, et al. Aortic reconstruction in kidney transplant recipients. Ann Vasc Surg. 1996;10(2):97-108.

13. Coosemans W, Nevelsteen A, Pirenne J, Vanrenterghem Y. Renal transplantation in patients with a vascular aortoiliac prosthesis. Transpl Proc. 1999;31(4):1925-1927.

Correspondencia autora: Dra. C. Aguilera Tubet

Servicio de Urología. Hospital Universitario Marqués de Valdecilla

Avda. de Valdecilla, s/n - 39008 Santander (Cantabria)

Tel.: 942202520

E-mail autora: car1677@hotmail.com

Información artículo: Original - Trasplante

Trabajo recibido: enero 2007

Trabajo aceptado: noviembre 2007 